\title{
ВЛИЯНИЕ ПРИЛИПАНИЯ НА ТЕРМОСТИМУЛИРОВАННУЮ РЕЛАКСАЦИЮ ПОВЕРХНОСТНОГО ПОТЕНЦИАЛА В МОНОПОЛЯРНЫХ ПОЛУПРОВОДНИКАХ
}

Расчет термостимулированной релаксации электроиндуцированного поверхностного потенциала (ТCP $\Delta U_{S}$ ) без учета прилипания носителей заряда в области пространственного заряда (ОПЗ) проведен в [1․ В настоящей работе рассмотрено влияние прилипания носителей в ОПЗ на TCP $\Delta U_{s}$. Анализ выполнен с помощью ЭBM.

\section{Постановка задачи}

Для конкретности рассмотрим монополярный полупроводник $n$-типа, на поверхности которого имеется неравновесный слой Шоттки, падение потенциала в котором обозначим через $\Delta U_{S}$. Такой слой можно создать приложением поперечного электрического поля, направленного против нормали к поверхности полупроводника [ $\left.{ }^{1}\right]$. Так как некоторая часть индуцированного отрицательного заряда захватывается на локальных уровнях, то после выключения поля поверхность остается в течение определенного времени заряженной отрицательно. При достаточно большой плотности поверхностного заряда вблизи поверхности образуется практически полностью истощенный свободными носителями слой. Релаксация $\Delta U_{S}$ происходит благодаря генерации свободных носителей заряда как на поверхности, так и в области положительного пространственного заряда. Примем во внимание два объемных уровня в ОПЗ и один поверхностный. Задавшись законом изменения температуры $T=T_{0}+\beta t$, систему уравнений, описывающих релаксацию в неравновесном слое Шоттки, можем представить в виде:

$$
\begin{aligned}
& j=q \mu n E, \\
& 1 / q \operatorname{div} j=-\left(\frac{\partial n}{\partial t}+\frac{\partial n_{1}}{\partial t}+\frac{\partial n_{2}}{\partial t}\right), \\
& \frac{\partial n_{1}}{\partial t}=\gamma_{1} n\left(N_{1}-n_{1}\right)-\gamma_{1} n_{1} N_{c} e^{-\frac{\varepsilon_{1}}{h T},} \\
& \frac{\partial n_{2}}{\partial t}=\gamma_{2} n\left(N_{2}-n_{2}\right)-\gamma_{2} n_{2} N_{c} e^{-\frac{\varepsilon_{2}}{h T}}, \\
& \frac{\partial E}{\partial x}=\frac{4 \pi}{x} \mathrm{Q} .
\end{aligned}
$$


Здесь $N_{1}, N_{2}$ и $n_{1}, n_{2}$ - концентрации локальных уровней и электронов на них в ОПЗ. Остальные обозначения общеприняты.

Плотность пространственного заряда @ в слое Шоттки можно записать в виде

$$
\varrho=q\left(N-n_{1}-n_{2}\right),
$$

где $N$ - нескомпенсированная глубокими акцепторами часть донорных примесей, заселенность которых меняется в процессе релаксации $\Delta U_{S}$. Величина $\Delta U_{\mathrm{S}}$ определяется из выражения

$$
\Delta U_{S}=-\int_{0}^{x} E d x-\frac{E_{0} L}{2},
$$

где $L-$ толщина слоя объемного заряда.

В выражении для тока в (1) пренебрегается диффузионной составляющей, так как она предполагается малой по сравнению с дрейфовой во всей ОПЗ, за исключением узкого слоя вблизи внутренней границы $(x \approx L)$.

Объединяя первые два уравнения системы (1) и вводя безразмерные величины

$$
\begin{array}{lcc}
x^{\prime}=\frac{x}{L_{0}}, \quad t^{\prime}=\frac{t}{t_{L}}, & T^{\prime}=\frac{1}{\beta t_{L}} T, \quad E^{\prime}=\frac{E \varkappa}{4 \pi \varrho_{0} L_{0}}, \\
n^{\prime}=\frac{n}{n_{0}}, & n^{\prime}{ }_{1}^{\prime}=\frac{n_{1}}{n_{0}}, & n^{\prime}{ }_{2}=\frac{n_{2}}{n_{0}}, \\
N^{\prime}{ }_{1}=\frac{N_{1}}{n_{0}}, & N^{\prime}=\frac{N_{2}}{n_{0}}, & N^{\prime}{ }_{c}=\frac{N_{c}}{n_{0}}, \\
\gamma^{\prime}{ }_{1}=\gamma_{1} n_{0} t_{L}, & \gamma^{\prime}{ }_{2}=\gamma_{2} n_{0} t_{L}, \quad n_{0}=\frac{\varrho_{0}}{q}, & \varepsilon^{\prime}=\frac{\varepsilon}{k T},
\end{array}
$$

где $t_{L}=\frac{x}{4 \pi \mu \varrho_{0}} \quad$ и $\varrho_{0}-$ плотность пространственного заряда в начале ТСР $\Delta U_{S}\left(T=T_{0}\right)$, получаем:

$$
\begin{aligned}
& E^{\prime} \frac{\partial n^{\prime}}{\partial x^{\prime}}-\frac{\partial n^{\prime}}{\partial T^{\prime}}=-\left(N^{\prime}-n_{1}^{\prime}-n_{2}^{\prime}-n^{\prime}\right) n^{\prime}+\gamma^{\prime}{ }_{1} n^{\prime}\left(N^{\prime}{ }_{1}-n^{\prime}{ }_{1}\right)- \\
& -\gamma_{1}^{\prime} n_{1}^{\prime} N_{c}^{\prime} e^{-\varepsilon^{\prime}}+\gamma_{2}^{\prime} n^{\prime}\left(N_{2}^{\prime}-n_{2}^{\prime}\right)-\gamma_{2}^{\prime} n^{\prime 2} N_{c}^{\prime} e^{-\varepsilon_{2}^{\prime}}, \\
& \frac{\partial n^{\prime}{ }_{1}}{\partial T^{\prime}}=\gamma^{\prime}{ }_{1} n^{\prime}\left(N^{\prime}{ }_{1}-n^{\prime}{ }_{1}\right)-\gamma^{\prime}{ }_{1} n^{\prime}{ }_{1} N_{c} e^{-\varepsilon^{\prime}{ }_{1}} \\
& \frac{\partial n_{2}^{\prime}}{\partial T^{\prime}}=\gamma_{2}^{\prime} n^{\prime}\left(N_{2}^{\prime}-n_{2}^{\prime}\right)-\gamma_{2}^{\prime} n_{2}^{\prime} N_{c}^{\prime} e^{-\varepsilon_{2}^{\prime}}, \\
& \frac{\partial E^{\prime}}{\partial x^{\prime}}=\left(N^{\prime}-n_{1}^{\prime}-n_{2}^{\prime}-n^{\prime}\right) \text {. }
\end{aligned}
$$

Для краткости обозначим правые части уравнений в системе (4) через $C_{1}, C_{2}, C_{3}$ и $C_{4}$ соответственно.

Первые три уравнения составляют гиперболическую систему квазилинейных дифференциальных уравнений. 
Для решения используем метод характеристик $\left[{ }^{2}\right]$. В данном случае на характеристиках, имеющих вид

$$
\frac{\mathrm{d} x^{\prime}}{\mathrm{d} T^{\prime}}=\lambda_{1}=-E^{\prime}, \quad \lambda_{2}=\lambda_{3}=0,
$$

выполняются соотношения

$$
\frac{\mathrm{d} n^{\prime}}{\mathrm{d} x^{\prime}}=\frac{C_{1}}{E^{\prime}}, \quad \frac{\mathrm{d} n_{1}^{\prime}}{\mathrm{d} T^{\prime}}=C_{2}, \quad \frac{\mathrm{d} n_{2}^{\prime}}{\mathrm{d} T^{\prime}}=C_{3} .
$$

Дополнительная формула для вычисления $E^{\prime}$ :

$$
\frac{\mathrm{d} E^{\prime}}{\mathrm{d} x^{\prime}}=C_{4}
$$

Для численного решения применим метод Массо, в основе которого лежит замена дифференциальных уравнений характеристик (5) и соотношений $(6)$, (7) соответствующими конечно-разностными уравнениями $\left[{ }^{2}\right]$. Сформулируем начальные и граничные условия для переменных $n^{\prime}, n_{1}^{\prime}, n_{2}^{\prime}, E^{\prime}$ в двух случаях генерации свободных носителей заряда: 1) в ОП3, 2) на поверхности. Примем, что при начальной температуре $T^{\prime}=T_{0}^{\prime}$ по всей ОПЗ $n^{\prime}=0$ и $\varrho_{0}=$ const.

В первом случае поверхностный заряд считается неизменным в процессе релаксации $\Delta U_{S}$, т. е. $E^{\prime}\left(0, T^{\prime}\right)=$ const. Предполагая, что при $x=0$ можно пренебречь прилипанием свободных электронов, мы придем к следующим граничным условиям для $n^{\prime}, n_{1}^{\prime}, n_{2}^{\prime}$ :

$n^{\prime}\left(0, T^{\prime}\right)=0$,

$n_{1}^{\prime}\left(0, T^{\prime}\right)=n_{1}^{\prime}\left(0, T^{\prime}{ }_{0}\right) \exp \left[\frac{\gamma_{1}^{\prime}}{\varepsilon_{1}^{\prime}} N_{c}^{\prime} e^{-\varepsilon^{\prime}}\left(\frac{1}{\varepsilon_{1}^{\prime}}-2\left(\frac{1}{\varepsilon_{1}^{\prime}}\right)^{2}+6\left(\frac{1}{\varepsilon_{1}^{\prime}}\right)^{3}\right)\right]$,

$n_{2}^{\prime}\left(0, T^{\prime}\right)=n_{2}^{\prime}\left(0, T_{0}^{\prime}\right) \exp \left[\frac{\gamma_{2}^{\prime}}{\varepsilon_{2}^{\prime}} N_{c}^{\prime} e^{-\varepsilon_{2}^{\prime}}\left(\frac{1}{\varepsilon_{2}^{\prime}}-?\left(\frac{1}{\varepsilon_{2}^{\prime}}\right)^{2}+6\left(\frac{1}{\varepsilon_{2}^{\prime}}\right)^{3}\right)\right]$.

Формулы для определения $n_{1}^{\prime}\left(0, T^{\prime}\right)$ и $n_{2}^{\prime}\left(0, T^{\prime}\right)$ получены из уравнений кинетики для соответствующих уровней $\left[{ }^{3}\right]$.

При генерации с поверхности граничное значение определяется мгновенным значением плотности поверхностного заряда. Предполагая отсутствие повторного прилипания электронов на поверхности, можно граничное условие для $E^{\prime}$, по аналогии с $n_{1,2}\left(0, T^{\prime}\right)$, задать в виде

$E^{\prime}\left(0, T^{\prime}\right)=E^{\prime}\left(0, T^{\prime}{ }_{0}\right) \exp \left[\frac{\gamma^{\prime}}{\varepsilon^{\prime}} N_{c}^{\prime} e^{-\varepsilon^{\prime}}\left(\frac{1}{\varepsilon^{\prime}}-2\left(\frac{1}{\varepsilon^{\prime}}\right)^{2}+6\left(\frac{1}{\varepsilon^{\prime}}\right)^{3}\right)\right]+E_{\text {ост }}^{\prime}$

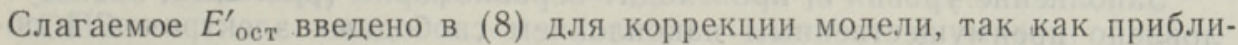
жение полностью истощенного слоя не выполняется при $U_{S} \rightarrow 0$ (т. е. и $E(0, T) \rightarrow 0)$. Это поле обусловлено остаточным зарядом на глубоких поверхностных уровнях или же равновесным поверхностным зарядом. Заряд этот в рассматриваемом процессе релаксации $\Delta U_{S}$ считается постоянным.

Во всех расчетах подвижность электронов в $c$-зоне считается не зависящей от температуры и равной $\mu=100 \mathrm{~cm}^{2} / 8 \cdot$ сек. Известно $\left[{ }^{4,5}\right]$, что подвижность $\mu$ может меняться в интересующем нас температурном интервале. Однако, так как целью расчетов является выяснение роли повторного прилипания на релаксацию $\Delta U_{\mathrm{S}}$, а не объяснение формы конкретных экспериментальных кривых ТCP $\Delta U_{S}$, подвижность принята 
постоянной. Сечения захвата $S$ предполагаются также не зависящими от температуры. Символ $S$ содержит и статистический весовой фактор примесного уровня.

Численные значения для эффективной плотности состояний и средней тепловой скорости электронов приняты при комнатной температуре равными $N_{c}(T)=2 \cdot 10^{18} \mathrm{cM}^{-3}$ и $v_{T}=10^{7} \mathrm{~cm} / \mathrm{ce \kappa}{ }^{-1}$. Глубина основного поверхностного или объемного уровня, определяющего релаксацию $\Delta U_{S}$, выбрана равной 0,25 эв, что примерно соответствует одному из экспериментально наблюдаемых значений в сульфиде кадмия [ $\left.{ }^{1}\right]$.

Расчет проведен в обоих случаях (поверхностная и объемная генерация) при двух соотношениях между временем жизни электрона относительно захвата на $i$-й уровень прилипания $\tau_{i}$ и временем пролета через ОПЗ $\tau_{l}, \tau_{l} \geqslant 10 \tau_{i}$ и $10 \tau_{l} \leqslant \tau_{i}$. Время пролета $\tau_{l}$ через слой Шоттки определяется выражением

$$
\tau_{l}=\frac{\varepsilon}{4 \pi \mu \varrho} \ln \frac{L_{0}}{L_{0}-x} .
$$

Поскольку это выражение логарифмически расходится при $x \rightarrow L_{0}$, то условимся за $\tau_{l}$ считать величину, получаемую из (9) при $x=0,99 L_{0}$.

\section{Результаты расчета и их обсуждение}

1. Объемная гене рация. Результаты расчета показаны на рис. $1, a$ и 2. Кривые 1 и 2 на рис. $1, a$ и сплошные кривые на рис. 2 относятся к случаю $\tau_{l} \geqslant 10 \tau_{2}$, а остальные - к случаю $10 \tau_{l} \leqslant \tau_{2}$. Величины всех постоянных, входящих в систему (1), даны в подписи к рис. 1. Цифры у кривых рис. 2 и 3 означают температуру в градусах Кельвина.

Учет повторного прилипания и заряда свободных электронов (рис. $1, a$; кривая 2) приводит в случае $\tau_{l} \geqslant 10 \tau_{2}$ к заметному смещению кривой TCP $\Delta U_{S}$ в сторону более высоких температур, а также к искажению ее формы.

Из рис. 2, $a$ видно, что в течение всего процесса релаксации концентрация свободных электронов не превышает $10^{4} \mathrm{~cm}^{-3}$. Поэтому их вкладом в $\mathrm{Q} / q \approx 10^{14} \mathrm{~cm}^{-3}$ можно пренебречь, как это было сделано в [ $\left.{ }^{1}\right]$. Следовательно, главной причиной смещения и искажения формы крнвой ТСР $\Delta U_{\mathrm{S}}$ должно являться прилипание электронов, освобождаемых термически с уровня $\varepsilon_{2}$.

Прилипание происходит на первоначально пустой уровень $\varepsilon_{1}$ и на $\varepsilon_{2}$. В данном примере сечение захвата электронов $S_{1}$ выбрано близким к типичному значению нейтральных центров $\left(5 \cdot 10^{-17} \mathrm{CM}^{2}\right)$.

Заполнение уровня $\varepsilon_{1}$ происходит неравномерно (рис. $\left.2, \sigma\right)$, что обусловлено преимущественным накоплением свободных электронов в области слабого поля (рис. 2,a). При этом в области наиболее крутого спада, как легко проверить подстановкой даңных рис. 2, a и 6 в уравнение кинетики для $n_{1}$, заполнение этого уровня существенно отличается от квазистационарного: захват на уровень $\varepsilon_{1}$ значительно превышает генерацию с него.

Сильная зависимость $n_{2}$ от $x$ в области $0 \leqslant x \leqslant 0,6 L$ в начальной стадии релаксации (рис. 2,8 ) свидетельствует о существенном повторном прилипании носителей заряда уже в первой половине ОПЗ, так как в противном случае концентрация должна была бы уменьшаться по $x$ равномерно. Во второй половине ОПЗ $(x \geqslant 0,6 L)$ вместо опустошения происходит дополнительное заполнение уровня $\varepsilon_{2}$. Это возможно 


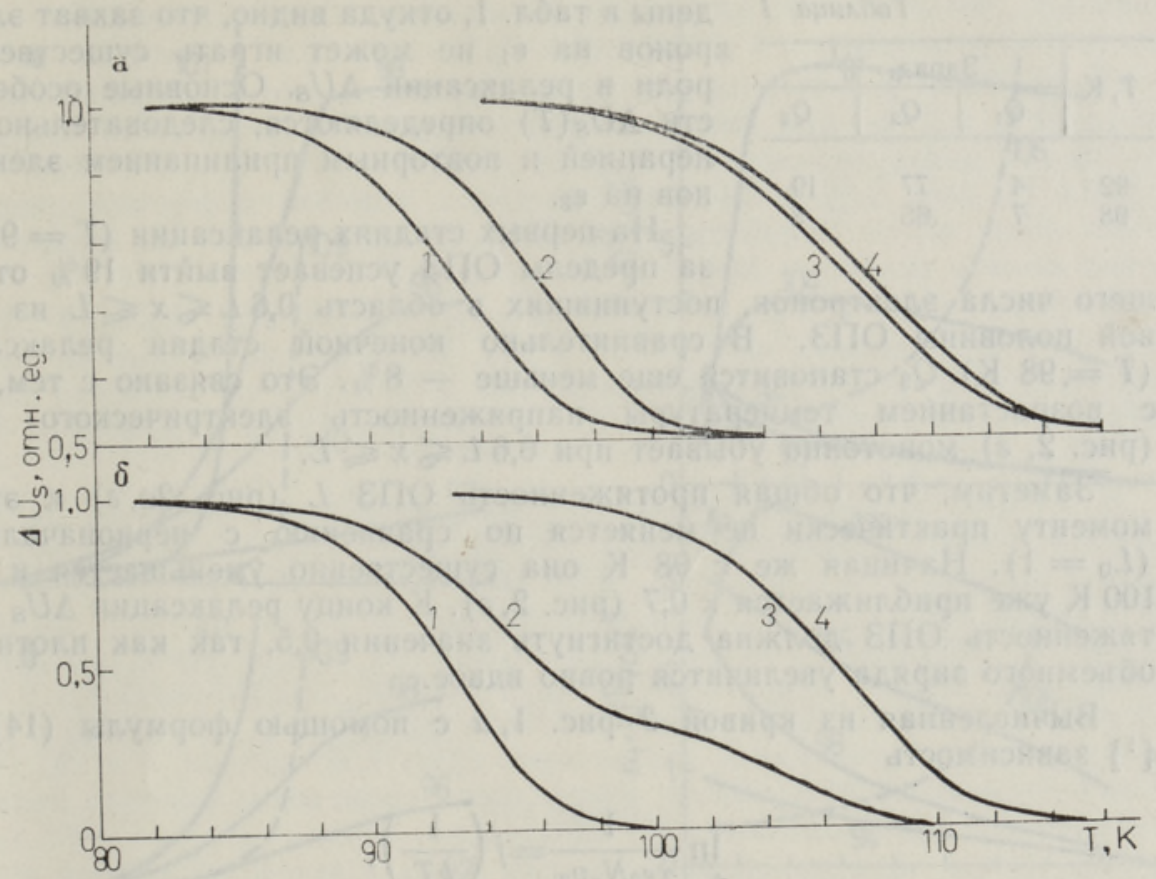

Рис. 1. Кривые ТСР $\Delta U_{S}(T)$, полученные с помощью ЭВМ, для объемной $(a)$ и поверхностной (б) генерации.

Общие параметры и константы при расчете:

$\varepsilon_{1}=0,22$ эв; $N_{1}=1 \cdot 10^{17} \quad \mathrm{CM}^{-3} ; n_{10} \cong 0 ; L_{0}=1 \cdot 10^{-3} \mathrm{cM} ;$

$N_{2}=1 \cdot 10^{16} ; \quad N_{c}=2 \cdot 10^{18} \mathrm{cM}^{-3} ; \quad x=12 ; \mu=100 \mathrm{cM}^{2} / \mathrm{s} \cdot$ сек;

$\mathrm{Q} / \mathrm{l}=1 \cdot 10^{14} \mathrm{CM}^{-3}$.

Значения варьируемых параметров:

$a-\varepsilon_{2}=0,25 \quad \exists B ; n_{20}=1 \cdot 10^{14} \quad M^{-3}$;

$1-S_{1}=0 ; S_{2}=5 \cdot 10^{-14} \mathrm{CM}^{2}$, без учета повторного прилипания;

$2-S_{1}=5 \cdot 10^{-17} \quad M^{2}, S_{2}=5 \cdot 10^{-14} \quad M^{2}$, с учетом повторного прилипания;

$3-S_{1}=0, S_{2}=5 \cdot 10^{-16} \mathrm{CM}^{2}$, без учета повторного прилипания;

$4-S_{1}=5 \cdot 10^{-17} \mathrm{CM}^{2}, S_{2}=5 \cdot 10^{-16} \mathrm{CM}^{2}$, с учетом повторного прилипания,

$\sigma-\varepsilon_{t s}=0,25 \ni 8, \varepsilon_{2}=0,27 \quad \ni 8, \quad n_{t s}=10^{11} \quad \mathrm{CM}^{-2}$;

$1-S_{1}=0, S_{2}=0, S_{t s}=5 \cdot 10^{-14} c^{2}$, без учета прилипания;

$2-S_{1}=5 \cdot 10^{-17} \mathrm{CM}^{2}, S_{2}=5 \cdot 10^{-14} \mathrm{CM}^{2}, S_{t s}=5 \cdot 10^{-14} \mathrm{CM}^{2}$, с учетом прилипа-

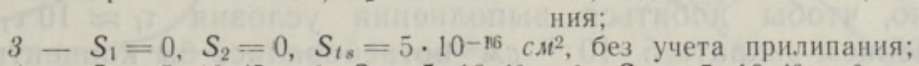

$4-S_{1}=5 \cdot 10^{-17} \mathrm{CM}^{2}, S_{2}=5 \cdot 10^{-16} \mathrm{cM}^{2}, S_{t s}=5 \cdot 10^{-16} \mathrm{cM}^{2}$, с учетом прилипания.

только благодаря электронам, выброшенным полем из первой половины ОПЗ.

На основе данных рис. $2, \sigma$ и в можно оценить долю общего заряда $Q$, освобожденного с уровня $\varepsilon_{2}$ в области $0 \leqslant x \leqslant 0,6 L$ к некоторому моменту времени. Ее составляют заряд электронов $Q_{1}$, захваченный на $\varepsilon_{1}$, и заряд $Q_{2}$, захваченный на $\varepsilon_{2}$ в области $0,6 L \leqslant x \leqslant L$. 3начения $Q$ и $Q_{2}$ определяются площадями между кривой $\left.n_{2}(x, T)\right|_{T=\text { const }}$ (рис. 2,8$)$ и уровнем $n_{2}=1 \cdot 10^{14} \mathrm{~cm}^{-3}$ в соответствующих интервалах $x$, а $Q_{1}$ - площадью между осью абсцисс и кривой $\left.n_{1}(x, T)\right|_{T=\text { const }}$.

Зная $Q, Q_{1}$ и $Q_{2}$ и пренебрегая концентрацией свободных электронов, можно определить заряд $Q_{3}$, вышедший к этому моменту за пределы ОПЗ.

Для двух температур значения $Q_{1}, Q_{2}$ и $Q_{3}$ в процентах от $Q$ приве- 
Таблица 1 дены в табл. 1, откуда видно, что захват элект-

\begin{tabular}{l|l|l|l}
\hline \multirow{2}{*}{$T, \mathrm{~K}$} & \multicolumn{4}{|c}{ Заряд, $\%$} \\
\cline { 2 - 4 } & $Q_{1}$ & $Q_{2}$ & $Q_{3}$ \\
\hline
\end{tabular}

$\begin{array}{rrrr}92 & 4 & 77 & 19 \\ 98 & 7 & 85 & 8\end{array}$

ронов на $\varepsilon_{1}$ не может играть существенной роли в релаксации $\Delta U_{S}$. Основные особенности $\Delta U_{S}(T)$ определяются, следовательно, генерацией и повторным прилипанием электронов на $\varepsilon_{2}$.

На первых стадиях релаксации $(T=92 \mathrm{~K})$

за пределы ОПЗ успевает выйти $19 \%$ от общего числа электронов, поступивших в область $0,6 L \leqslant x \leqslant L$ из первой половины ОПЗ. В сравнительно конечной стадии релаксации $(T=98 \mathrm{~K}) Q_{3}$ становится еще меньше - $8 \%$. Это связано с тем, что с возрастанием температуры напряженность электрического поля (рис. 2, г) монотонно убывает при $0,6 L \leqslant x \leqslant L$.

Заметим, что общая протяженность ОПЗ $L$ (рис. 28,2 ) к этому моменту практически не меняется по сравнению с первоначальной $\left(L_{0}=1\right)$. Начиная же с $98 \mathrm{~K}$ она существенно уменьшается и при $100 \mathrm{~K}$ уже приближается к 0,7 (рис. 2 , г). K концу релаксации $\Delta U_{\mathrm{S}}$ протяженность ОПЗ должна достигнуть значения 0,5, так как плотность объемного заряда увеличится ровно вдвое.

Вычисленная из кривой 2 рис. $1, a$ с помощью формулы (14) из [ $\left.{ }^{1}\right]$ зависимость

$$
\ln \frac{1}{\tau \varepsilon_{2} N_{c} v_{T}}=f\left(\frac{1}{k T}\right),
$$

где $\tau-$ время жизни электрона на уровне $\varepsilon_{2}$, не является линейной. Однако, аппроксимируя ее отдельные участки прямыми, можем оценить $\varepsilon_{2}$. Так, например, использовав участок $\Delta U_{S}(T)$ в интервале $90-$ $92 \mathrm{~K}$, получим $\varepsilon_{2}=0,234$ эв, а в интервале $94-96 \mathrm{~K}-\varepsilon_{2}=0,28$ эв. Сечения захвата будут соответственно $2 \cdot 10^{-15}$ и $8 \cdot 10^{-13}$ c. $^{2}$ (вместо $5 \cdot 10^{-14}\left(\mu^{2}\right)$. Как видно, полученные значения $\varepsilon_{2}$ и $S_{2}$ отличаются от истинных гриблизительно на $10 \%$.

В случае монокристаллов сульфида кадмия условие $\tau_{l} \geqslant 10 \tau_{i}$ будет выполняться лишь для центров с предельно большими сечениями захвата $\left(\sim 10^{-13}-10^{-14} c \mu^{2}\right)$, причем при наличии довольно значительных концентраций не занятых электронами центров $\left(>1 \cdot 10^{16} \mathrm{~cm}^{3}\right)$. В данном примере $\tau_{l} \approx 3 \cdot 10^{-9}$ сек, $\tau_{2} \approx 2 \cdot 10^{-10}$ сек, а $\tau_{1} \approx 2 \cdot 10^{-8}$ сек. Следовательно, чтобы добиться выполнения условия $\tau_{l} \approx 10 \tau_{1}$, для центров с сечением захвата $5 \cdot 10^{-17} \mathrm{~cm}^{2}$ потребовалась бы концентрация $10^{19} \mathrm{CM}^{-3}$.

Уменьшение сечения захвата центров $\varepsilon_{2}$ на два порядка ( $\tau_{2} \approx$ $\approx 2 \cdot 10^{-8}(е \kappa)$, при сохранении значений всех остальных параметров модели, позволяет реализовать случай $\tau_{2} \geqslant 10 \tau_{l}$. Кривые 3 и 4 релаксации $\Delta U_{S}$ (рис. $\left.1, a\right)$ смещаются при этом на $14 \mathrm{~K}$ в сторону более высоких температур, а сдвиг, обусловленный прилипанием, уменьшается значительно. Распределения $n(x), n_{1}(x), n_{2}(x)$ и $E(x)$ для температуры $108 \mathrm{~K}$, при которой зависимости этих величин от $x$ наиболее сильно выражены, показаны на рис. 2 пунктирными кривыми. Мы видим, что в большей части ОПЗ учет концентраций $n(x), n_{1}(x)$ и $n_{2}(x)$ не дает существенного вклада в плотность пространственного заряда, которую можно считать практически постоянной. Заметное изменение плотности пространственного заряда имеет место лишь около границы ОПЗ. Однако это изменение сказывается на $\Delta U_{S}$ довольно слабо, так как в этой области электрическое поле мало. Значения $S_{2}$ и $\varepsilon_{2}$, определенные по температурной зависимости времени жизни электрона на 

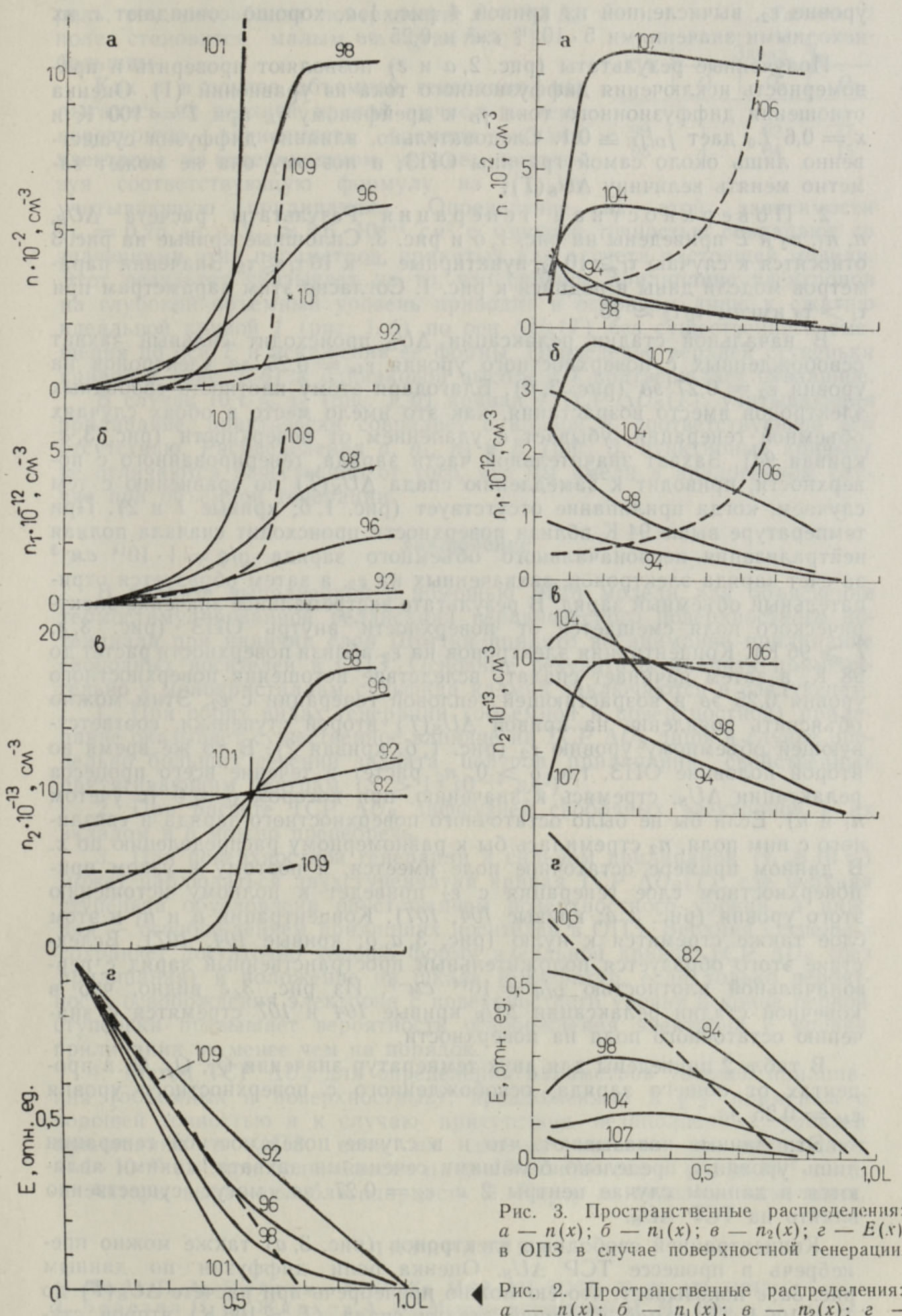

Рис. 3. Пространственные распределения $a-n(x) ; \sigma-n_{1}(x) ; \varepsilon-n_{2}(x) ; c-E(x)$ в ОПЗ в случае поверхностной генерацин.

4 Рис. 2. Пространственные распределения: $a-n(x) ; \sigma-n_{1}(x) ; \quad$ в $-n_{2}(x) ; 2-$ $E(x)$ в ОПЗ в случае объемной генерации. 
уровне $\varepsilon_{2}$, вычисленной из кривой 4 рис. $1, a$, хорошо совпадают с их исходными значениями $5 \cdot 10^{-16} \mathrm{CM}^{2}$ и 0,25 эв.

Полученные результаты (рис. $2, a$ и г) позволяют проверить и правомерность исключения диффузионного тока из уравнений (1). Оценка отношения диффузионного тока $j_{D}$ к дрейфовому $j_{E}$ при $T=100 \mathrm{~K}$ и $x=0,6 L_{0}$ дает $j_{D} / j_{E} \cong 0,1$. Следовательно, влияние диффузии существенно лишь около самой границы ОПЗ, и поэтому она не может заметно менять величину $\Delta U_{\mathrm{S}}(T)$.

2. Поверхностная генерация Результаты расчета $\Delta U_{S}$, $n, n_{1}, n_{2}$ и $E$ приведены на рис. $1, \sigma$ и рис. 3. Сплошные кривые на рис. 3 относятся к случаю $\tau_{l} \geqslant 10 \tau_{2}$, пунктирные - к $10 \tau_{l} \leqslant \tau_{2}$. Значения параметров модели даны в подписи к рис. 1. Согласно этим параметрам при $\tau_{l}>\tau_{2}$ имеем $\tau_{l} / \tau_{2} \approx 15$.

В начальной стадии релаксации $\Delta U_{S}$ происходит сильный захват освобожденных с поверхностного уровня $\varepsilon_{t s}=0,25$ эв электронов на уровни $\varepsilon_{2}=0,27$ эв (рис. $\left.3, \sigma\right)$. Благодаря этому плотность свободных электронов вместо возрастания, как это имело место в обоих случаях объемной генерации, убывает с удалением от поверхности (рис. $3, a$; кривая 94). Захват значительной части заряда, генерированного с поверхности, приводит к замедлению спада $\Delta U_{S}(T)$ по сравнению с тем случаем, когда прилипание отсутствует (рис. 1, б; кривые 1 и 2). При температуре выше $94 \mathrm{~K}$ вблизи поверхности происходит сначала полная нейтрализация первоначального объемного заряда $\mathrm{g} / \mathrm{q}=1 \cdot 10^{14} \mathrm{~cm}^{-3}$ за счет заряда электронов, захваченных на $\varepsilon_{2}$, а затем образуется отрицательный объемный заряд. В результате экстремальное значение электрического поля смещается от поверхности внутрь ОПЗ (рис. 3, г; $T \geqslant 96 \mathrm{~K})$. Концентрация электронов на $\varepsilon_{2}$ вблизи поверхности растет до $98 \mathrm{~K}$, а затем начинает спадать вследствие истощения поверхностного уровня 0,25 эв и возрастающей тепловой генерации с $\varepsilon_{2}$. Этим можно объяснить появление на кривой $\Delta U_{S}(T)$ второй ступеньки, соответствующей объемному уровню $\varepsilon_{2}$ (рис. 1, б, кривая 2). В то же время во второй половине ОПЗ, где $\varrho>0, n_{2}$ растет в течение всего процесса релаксации $\Delta U_{S}$, стремясь к значению, при котором $\mathrm{g}=0$ (с учетом $n_{1}$ и $\left.n\right)$. Если бы не было остаточного поверхностного заряда и связанного с ним поля, $n_{2}$ стремилась бы к равномерному распределению по $x$. В данном примере остаточное поле имеется, и поэтому в узком приповерхностном слое генерация с $\varepsilon_{2}$ приведет к полному истощению этого уровня (рис. 3,8 ; кривые 104,107 ). Концентрации $n$ и $n_{1}$ в этом слое также стремятся к нулю (рис. 3, а, б; кривые 104, 107). Вследствие этого образуется положительный пространственный заряд с первоначальной плотностью $\mathrm{g} / q=10^{14} \mathrm{~cm}^{-3}$. Из рис. 3,2 видно, что в конечной стадии релаксации $\Delta U_{\mathrm{S}}$ кривые 104 и 107 стремятся к значению остаточного поля на поверхности.

В табл. 2 приведены для двух температур значения $Q_{1}, Q_{2}, Q_{3}$ в процентах от общего заряда, освобожденного с поверхностного уровня $\varepsilon_{t s}=0,25$ эв.

Эти данные показывают, что и в случае поверхностной генерации лишь уровни с предельно большими сечениями захвата, какими являются в данном случае центры 2 с $\varepsilon_{2}=0,27$ эв, могут существенно влиять на ТCP $\Delta U_{\mathrm{S}}$.

Концентрацией свободных электронов (рис. 3, a) также можно пренебречь в процессе TCP $\Delta U_{S}$. Оценка роли диффузии по данным рис. $3 a$, г показывает, что ею можно пренебречь при расчете $\Delta U_{B}(T)$ до $T=106 \mathrm{~K}$. В самой конечной стадии спада $(T>106 \mathrm{~K})$ второй ступеньки $\Delta U_{S}(T)$ (рис. 1, б, кривая 2) диффузия может играть заметную 
роль, так как вблизи поверхности $x \approx 0,1 L_{0}$ поле становится малым, a $\operatorname{grad} n(x, T)-$ большим.

Как и в случае объемной генерации, мы пытались из верхней кривой вычислить температурную зависимость времени жизни электрона на поверхностном уровне, используя соответствующую формулу из $\left[{ }^{1}\right]$, не учитывающую прилипания. Определение из этой зависимости $\varepsilon_{t s}=0,25$ эв и $S_{t s} \cong 5,6 \cdot 10^{-14} c \mu^{2}$ с хорошей точностью совпадают со значениями этих параметров, принятых для расчета настоящей модели. Этот результат указывает на то, что сильное прилипание электронов на глубокий объемный уровень приводит в основном лишь к сжатию идеальной кривой 1 (рис. $1, \sigma)$ по оси $\Delta U_{\mathrm{S}}(T)$ без существенного смещения по оси $T$ и искажения ее формы. Приведение верхней ступеньки кривой 2 (рис. 1,6$)$ к одинаковой высоте с 1 подтверждает сказанное.

В случае $10 \tau_{l} \leqslant \tau_{2}$ (рис. $\left.1, \sigma\right)$ кривая 4 TCP $\Delta U_{S}(T)$, учитывающая прилипание, практически совпадает с кривой 3 , соответствующей его отсутствию. Распределения $n, n_{1}, n_{2}$ и $E$ (рис. 3 , пунктирные кривые) не отличаются качественно от того, что мы имели в аналогичном случае при объемной генерации.

\section{Заключение}

В анализе выполненной с помощью ЭВМ модели для объяснения термостимулированной релаксации неравновесного поверхностного потенциала при наличии слоя Шоттки принято во внимание прилипание свободных носителей в ОПЗ. Основные результаты анализа применительно к монокристаллам сульфида кадмия заключаются в следующем.

1. При обоих видах генерации (объемной и поверхностной) прилипание оказывает существенное влияние на TCP $\Delta U_{S}$ лишь в случае предельно больших сечений захвата центров прилипания, свойственных прутягивающим центрам $\left(S>1 \cdot 10^{-14} c M^{2}\right)$.

2. При расчете ТСР $\Delta U_{S}$ диффузней свободных электронов и их вкладом в е можно пренебречь.

3. При поверхностной генерации и сильном прилипании $\left(\tau_{l} \geqslant 10 \tau_{2}\right)$ может наблюдаться двухступенчатая кривая ТCP $\Delta U_{S}(T)$, где верхняя ступенька обусловлена опустошением поверхностного уровня, а нижняя - освобождением прилипших носителей в ОПЗ. Верхняя стипенька сохраняет достаточно хорошо форму идеальной кривой TCP $\Delta U_{\mathrm{S}}$ (без прилипания) и положение на шкале температур, если только вероятность освобождения электрона с поверхностного уровня в конце первой ступеньки превышает вероятность освобождения электрона с уровня прилипания не менее чем на порядок.

4. Метод TCP $\Delta U_{\mathrm{S}}$ для определения параметров уровней прилипания (объемных и поверхностных), предложенный в $\left[{ }^{1}\right]$, применим с хорошей точностью и к случаю присутствия незаполненных уровней прилипания в ОПЗ, если их сечения захвата не превышают $\sim 1 \cdot 10^{-15} c \mu^{2}$ при подвижности $\mu_{n} \geqslant 100 \mathrm{~cm}^{2} / 8 \cdot$ сек и $\mathrm{g} / q \geqslant 10^{14} \cdot \mathrm{cm}^{-3}$.

Авторы выражают благодарность Я. Кирсу за полезные замечания.

\section{Л И Т Е А Т Р Р А}

1. Л уук П. А., Пи йльм а М. Р.. Тр. ИФА АН ЭССР, № 41, 171 (1972).

2. Березин И. С., Жидков А. И., Методы вычислений II, М., 1966.

3. Актуальные вопросы физики полупроводников и полупроводниковых приборов, Вильнюс, 1969 , с. 153. 
4. Ш ейнкман $M$. К., К орсунская Н. Е., М а ркевич И. В., УФК, 8,747 (1969); Т рофименко А. П., Федорус Г. А., Шейнкман М. К., ФТТ, $5,1805\left(1963^{3}\right)$.

5. Li pskis K., S a k a l a s A., V i šč a k a s J., Phys. stat. sol. (a), 2, 225 (1970).

Ннститут физики

Академии наук Эстонской ССР
Поступила в редакцию 29/IV 1974

J. BERGMANN, P. LOUK, A. RAIDARU

\section{HAARDUMISE MOJU PINNAPOTENTSIAALI TERMOSTIMULEERITUD RELAKSATSIOONILE MONOPOLAARSETES POOLJUHTIDES}

Käsitletakse laengukandjate haardumist arvestavat mudelit mittetasakaalulise pinnapotentsiaali termostimuleeritud relaksatsiooni kirjeldamiseks Schottky kihi korral. Analüüs on tehtud raali abil. Vaadeldakse tugeva ja nōrga haardumise juhtu, kui vabu laengukandjaid genereeritakse kas pinnal vōi ruumlaengu piirkonnas.

\section{J. BERGMANN, P. LOUK, A. RAIDARU}

\section{INFLUENCE OF TRAPPING ON THERMALLY STIMULATED RELAXATION OF THE SURFACE POTENTIAL IN UNIPOLAR SEMICONDUCTORS}

A model for thermally stimulated relaxation of the nonequilibrium surface potential has been treated. The numerical analysis by means of a computer accounts for free carrier trapping in the depletion type surface space charge region. The surface potential decay has been considered in the cases of strong and weak trapping and by free carrier generation from the local states in the space charge region or on the surface. 